\title{
Prevalencia y naturaleza de los eventos adversos en hospitales de Venezuela.
}

\author{
Mónica Susana Chirinos Muños', Carola Viviana Orrego Villagran ${ }^{2}$, \\ César Alberto Montoya Medero y Rosa Suñol Sala \\ ${ }^{1}$ Programa de Administración del Sector Salud-Universidad del Zulia, Maracaibo- \\ Venezuela. Programa de Doctorado de la Universitat Autónoma de Barcelona-España. \\ ${ }^{2}$ Instituto Universitario Avedis Donabedian, Barcelona-Spain. Research Network \\ on Health Service Chronic Diseases REDISSEC. Barcelona, Spain. \\ ${ }^{3}$ Instituto Universitario de Tecnología de Maracaibo. Edo. Zulia, Venezuela.
}

Palabras clave: seguridad del paciente; errores médicos; efectos adversos; epidemiología.

Resumen. La falta de seguridad del paciente es un problema de salud pública que ocasiona daños y la mayoría son prevenibles. Por ello, se determinó la prevalencia y se describieron los elementos epidemiológicos de los Eventos Adversos (EA) detectados en hospitales del estado Zulia. Es un estudio de corte transeccional y descriptivo en cuatro hospitales (dos públicos y dos privados), basado en la revisión de historias clínicas, y la observación de 556 pacientes hospitalizados, seleccionados aleatoriamente en cada hospital. Se aplicó una guía de cribado de eventos adversos, un formulario resumen y un cuestionario modular para la revisión de casos (MRF2). Como resultados, del total de pacientes estudiados, 93 presentaron al menos un EA, de los cuales más del 80\% se consideraron evitables. La tasa global de EA fue del 16,54\% (IC del 95\%: 15,98 - 17,01), con un $20 \%(\mathrm{IC}=95 \% 19,1-21)$, en los hospitales públicos y un 18\% en los hospitales privados ( $\mathrm{IC}=95 \% 17,1-19)$, sin diferencia estadística signnificativa de acuerdo al tipo de hospital $(\mathrm{p}=0,11)$. No se detectaron casos de muertes consecuencia de algún EA, sin embargó, el 29,3\% de la población, presentó un grado severo de incapacidad física consecuencia de los EA de gravedad alta. Finalmente, de este estudio se deduce que, por cada 20 pacientes hospitalizados en cada una de las instituciones, podrían ocurrir 1,7 EA; lo que obliga a un llamado urǵente a priorizar las políticas y estrategias en pro de la investigación, documentación y mejoría de la seguridad del paciente en la reǵión, tanto en hospitales públicos como privados.

Autor de Correspondencia: Mónica Susana Chirinos Muñoz. Programa de Administración del Sector Salud-Universidad del Zulia, Maracaibo-Venezuela. Correo electrónico: monicasuchm@yahoo.com. 


\title{
Prevalence and nature of adverse events in hospitals in Venezuela.
}

Invest Clin 2019; 60 (4): 296-309

Keys words: patient safety; medical errors; adverse effects; epidemiology.

\begin{abstract}
Patient safety is a public health problem, causing damage to patients, the majority preventable. Therefore, the aim is to determine the prevalence and describe elements of adverse events (AE) detected in four hospitals in Zulia state, Venezuela. Cross-sectional study in four hospitals (two public and two private) based on review of the clinical records, and the observation of 556 hospitalized patients, randomly selected during one month of study in each hospital. The screening guide based on the IBEAS study, a summary form and the modular questionnaire for case review were applied. Of the total of patients studied, 93 had at least one $\mathrm{AE}$. The global AE prevalence was 16.5\% (95\% CI $15,98-17,01$, of which $81.7 \%$ were considered avoidable. In public and private hospitals, $\mathrm{AE}$ prevalences were 20\% ( $\mathrm{IC}=95 \% 19,1-21)$ and 18\% (IC=95\% $17,1-19$.$) , respectively, without statistically significant difference (p=0.11)$. There were no deaths as a result of some AE, however $29.3 \%$ of the population presented a severe degree of physical disability due to high severity AEs. In four hospitals of the Zulia state in Venezuela, 1.7 AEs occur for every 20 treated patients. We recommend an urgent call for prioritization of policies and strategies in favor of research, documentation and improvement of patient safety in the regiion, both in public and private hospitals.
\end{abstract}

Recibido: 15-02-2019 Aceptado: 19-09-2019

\section{INTRODUCCIÓN}

La seguridad del paciente ha ganado numerosos espacios de disertación y reflexión, especialmente después de la publicación "To err is human" (1), la cual estableció las bases para la sensibilización ante este tema y en consecuencia para dinamizar un movimiento mundial de esfuerzos en la formulación de planes, iniciativas y acciones en pro de la mejora y garantizar seguridad en la atención en salud (2-4). En esa dirección, la investigación representa un esfuerzo al nivel primario, para dinamizar los cambios necesarios, ya que se identifican los problemas como oportunidades de mejora y se planifica su abordaje con mayor conciencia $(5,6)$. En este sentido, se han realizado a lo largo de muchos años y hasta la actualidad, numerosas iniciativas investigativas para conocer la frecuencia de los Eventos Adversos (EA) (716) y reiterar la importancia de este tema en razón de los alarmantes resultados.

Ese esfuerzo, también se refleja en Latinoamérica, donde se ha enriquecido el conocimiento sobre la frecuencia de los EA; en este sentido el estudio IBEAS (Iberoamerican Study of Adverse Effects) $(17,18)$ despertó el interés por la seguridad del paciente y sus resultados impulsaron estrategias en la región dinamizando proyectos y resoluciones que dan garantía de una atención sanitaria más segura (19-21). Este estudio obtuvo una prevalencia global de pacientes

Vol. 60(4): 296 - 309, 2019 
con algún EA de un $10,5 \%$, con un resultado que va desde un $7,7 \%(\mathrm{IC}=6,8-8,7)$ para el país con menor evaluación, hasta un $13,1 \%$ $(\mathrm{IC}=11,8-14,5)$; siendo una recomendación global de este tipo de estudios, realizar investigaciones con mayor especificidad en cada país, como inclusive se ha hecho;(23) materializándolo en este caso en la Región Zulia en Venezuela.

Actualmente en Venezuela, la situación referente a la seguridad del paciente, como política de salud se encuentra en fase incipiente, sin concreción operativa, aunque se presentan algunas iniciativas interesantes (24-26). La falta de información documentada suficiente y objetiva sobre este tema, dificulta el impulso en la toma de decisiones, aunada a una crisis del sector salud que afecta tanto la gestión pública como a la privada.

Esta investigación inédita en el país, se desarrolló en el estado Zulia, por ser la región más poblada del territorio nacional, y el quinto de mayor superficie en Venezuela (27); planteándose como objetivo la identificación de la prevalencia y los elementos epidemiológicos asociados a la aparición y caracterización de eventos adversos (EA), en hospitales de mediana-alta complejidad, en el que se valoró desde una perspectiva comparativa hospitales públicos y hospitales privados $(17,28,29)$.

\section{MATERIAL Y MÉTODOS}

El presente estudio es epidemiológico de corte descriptivo con un diseño transeccional-observacional. Para identificar los EA, se utilizó la definición aplicada en el estudio IBEAS $(17,18)$, así como en la mayoría de los estudios epidemiológicos donde los EA están definidos como: "Todo accidente imprevisto e inesperado recogido en la historia clínica que ha causado muerte, lesión y/o incapacidad y/o prolongación de la estancia, que se deriva de la asistencia sanitaria más que de la enfermedad de base del paciente".

Para recabar la información necesaria, durante el período establecido entre abril y julio de 2015, se consideró como población a los hospitales de mediana-alta complejidad del Estado Zulia (Tipo III y IV) (30), los cuales representan un conjunto de 13 hospitales en la región. En razón de su dispersión geográfica se empleó el muestreo polietápico de conglomerados con estratificación (31), procediendo de la siguiente manera: I.- En una primera etapa se dividió el estado Zulia en dos Unidades Primarias de Muestreo (UPM): a.- la Costa Oriental del Lago (Municipio Cabimas), contentivo de 2 hospitales, de donde se seleccionó al azar 1 hospital y b.- la Costa Occidental del Lago (Municipio Maracaibo) con 11 hospitales, donde se seleccionaron 3 al azar, representando fracciones muéstrales del 50 y $31 \%$, respectivamente. II.- Unidades Secundarias de Muestreo: En de los hospitales establecidos para el estudio, se calculó el total de pacientes a estudiar, mediante la aplicación de la fórmula de las proporciones para un número finito de individuos $(\mathrm{N}=118)$, calculado de acuerdo al número total de admisiones hospitalarias mensuales de pacientes $(\mathrm{N}=3000)$, los cuales atendían ciertos criterios de inclusión en el estudio: *casos con un mínimo de 72 horas desde el ingreso en las salas de hospitalización, "Pacientes admitidos en salas de hospitalización de las Unidades asistenciales de Medicina Interna, Cirugía General, Traumatología y Ortopedia, Gastroenterología, Nefro-Urología y Pediatría. En cada hospital los pacientes fueron seleccionados secuencialmente al azar durante 30 días, hasta alcanzar como mínimo el número estimado, lográndose en el período propuesto muestrear 139 por hospital, con un total de 556 pacientes en los 4 hospitales.

Se consideraron los EA en cualquiera de los siguientes momentos: a.- El EA ocurrió durante el ingreso a la sala de hospitalización, pero fue identificado durante el período de estudio.b.- El EA ocurrió durante la estancia hospitalaria y se detectó en cualquier momento de la hospitalización.c.- El EA ocurrió culminada la estancia hospitalaria, y se detectó durante al alta. 
Las variables principales estudiadas fueron: 1. Prevalencia del EA 2. Gravedad del EA; 3. Tipo de hospital donde ocurrió el EA; 4. Naturaleza o Tipo de EA, relacionada con cuidados, diaǵnóstico, medicación, procedimiento, infección nosocomial, mixta, otros; 5. Posibilidad de Prevención (Evitabilidad) del EA; 6. Factores de riesgo en la ocurrencia del EA; 7. Período de hospitalización donde ocurrió el EA; 8. Repercusión del EA en la estancia; 9. Incapacidad provocada por el EA. Se capacitaron encuestadores y revisores, como garantía de homogeneidad del manejo de la muestra que incluyó un simulacro personal asistencial-paciente, y una prueba piloto con una muestra al azar de 20 pacientes, durante 10 días; se utilizaron métodos de vigilancia activa a través de la observación de pacientes, revisión de historias clínicas y afianzando conceptos entre revisores.

\section{Los instrumentos utilizados se descri- ben a continuación:}

1. Guía para el cribado de EA y formulario resumen de la historia clínica, adaptada del proyecto IDEA (32). Basado en la experiencia de adecuación realizada en el estudio IBEAS, se agregaron al listado cinco factores de riesgo intrínsecos: hiperuricemia, enfermedad cardiovascular (ECV), enfermedad vascular periférica, tabaquismo y alcoholismo. La historia fue recogida por cuatro médicos con experiencia clínica mayor de cinco años, que no presentaron compromisos laborales con ninguna institución del estudio (Revisor Primario).

2. Versión española del cuestionario modular para revisión retrospectiva de casos MRF2 (Modular Revision Form), modificado (Proyecto IDEA) (33). Aplicada por un encuestador y analizada por un médico residente del 4to año de la especialidad que cursase, verificada posteriormente, por un especialista. De existir discrepancia se discutió el caso con un tercer médico, identificado como especialista invitado previamente capacitado en el tema.
Fases de Aplicación y análisis de los instrumentos:

- Fase 1: Cribado y caracterización de EA: Aplicación de la Guía de Cribado y revisión de historias clínicas con el uso de formulario resumen e identificación de ausencia o presencia de EA. Si se identificaba la presencia de EA, se pasaba a la Fase 2, de lo contrario culminaba el análisis.

- Fase 2: Aplicación del MRF2, posterior al alta del paciente.

- Fase 3: Valoración del MRF2 por el especialista, y validación de la concordancia. Si no había concordancia pasaba a la Fase 4 .

- Fase 4: Discusión final con revisores primarios y especialista invitado; confirmación de las fases 2 y 3 a través de la estadística de estudio; si existiese incongruencia, se descartaba el caso como parte de la prevalencia.

\section{Análisis de Datos:}

Para describir la naturaleza de los EA, mediante los datos recogidos por los instrumentos de medición, se emplearon distribuciones de frecuencias y estadísticos descriptivos como la media y la desviación estándar.

Con el objeto de profundizar en la descripción, se empleó la prueba de Chi Cuadrado $(\chi 2)$ para determinar la existencia de diferencias estadísticas entre las frecuencias de ocurrencia de las variables empleadas para realizar la caracterización: "Posibilidad de prevención del EA" "tipo del EA", "Período de hospitalización donde ocurrió el EA", "Niveles de gravedad", "Repercusión del EA en la estancia", "Incapacidad provocada por el EA" y se discriminó según el tipo de hospital: HPR (Hospitales Privados) y HP (Hospitales Públicos). El análisis de correspondencia permitió precisar las celdas donde ocurrió la asociación.

\section{Consideraciones éticas:}

El protocolo de estudio fue validado por el Comité de Ética de cada institución incluida en el estudio. 


\section{RESULTADOS}

Cribado de los Eventos Adversos:

Se realizó cribado y aplicación del formulario resumen, al 100\% $(\mathrm{N}=556)$ de los pacientes seleccionados y hospitalizados; de esta cifra el $31,8 \%(\mathrm{~N}=177)$ fueron cribados positivos por fallos en la seguridad del paciente, para posterior revisión de historia clínica. De estos casos se descartó el 1\% $(\mathrm{N}=6)$, debido a que la calidad de la historia clínica era deficiente para continuar con el análisis (Fig. 1); el 4,6\% $(\mathrm{N}=26)$ fueron falsos positivos y el 25\% ( $\mathrm{N}=139)$ tuvieron alguna lesión identificada; de los cuales $16,7 \%(\mathrm{~N}=93)$ de los casos se relacionaron con daño al paciente asociado a la atención en salud, siendo esta la prevalencia global de EA, ubicado el $9,7 \%(\mathrm{~N}=54)(\mathrm{p}=54 / 263=20 \% \mathrm{E}, \mathrm{E}: 2,6 \mathrm{I} / \mathrm{C}$ : 99\%) en hospitales públicos y el 7\% (39) en hospitales privados $(\mathrm{p}=39 / 214=18 \% \mathrm{E}, \mathrm{E}$ : 1,4 I/C: 95\%), que posterior a un ajuste entre hospitales se detectó, que no fue significativa la diferencia entre hospitales.

\section{Caracterización de la muestra estudiada:}

Tal como se observa en la Tabla I, con respecto a la edad, tanto en los hospitales públicos (HP) como hospitales privados (HPR), la población mayormente representada presenta un rango de edad de 26 a 45 años (46\%, muy cercana a la mediana), seguido del rango entre los 46 a 65 años.

Referente al nivel educativo, en los HP sobresalieron los pacientes con un nivel inferior de educación (Analfabetas-primaria) representando el 53,2\% en contraposición con los HPR, donde destacó la presencia del nivel educativo superior (Técnicos/UniversitarioUniversitarios Superiores) constituyendo el $38,8 \%$ (Tabla I).

\section{Caracterización de los Eventos Adversos:}

Tal como se evidencia en la Tabla II, se presentan los resultados de la caracterización de los EA, valorando ciertos criterios que se describen a continuación:

Posibilidad de prevención del EA: en este sentido los revisores detectaron una tasa elevada de EA prevenibles, es decir que pudieron haberse evitado, representando en los públicos el $75.5 \%$ de los EA detectados mientras que en los en los privados el 82,5\% $(\mathrm{p} \leq 0,05)$.

Referente a los tipos de EA detectados, de acuerdo a la Tabla II, no existe predominio de un determinado tipo de EA en alguno de los hospitales estudiados $(\mathrm{p}>0,05)$ (Tabla II). La incidencia porcentual de cada uno de las posibles causas de EA se presenta a continuación en orden descendente: Cuidados $(30,70 \%)$, Mixtos (26,95\%), Infección Noso-

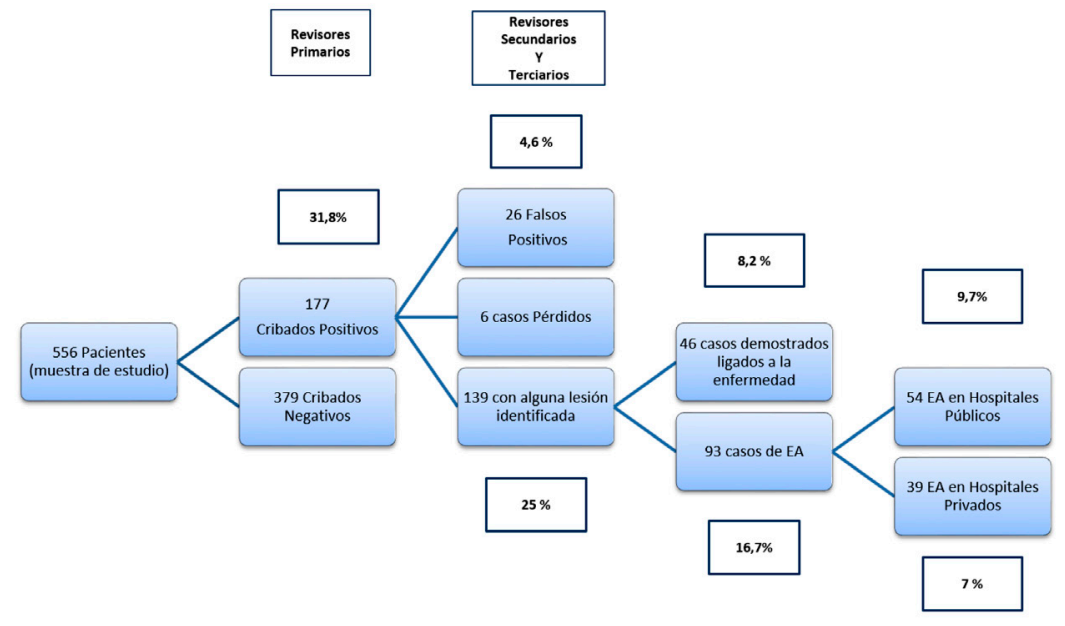

Fig. 1. Determinación y cribado de los eventos adversos. 
TABLA I

CARACTERIZAGIÓN DE LA MUESTRA ESTUDIADA EN LAS INSTITUCIONES DE SALUD PÚBLICAS Y PRIVADAS EVALUADAS.

\begin{tabular}{|c|c|c|c|c|c|}
\hline \multirow[t]{2}{*}{ Variables } & \multicolumn{2}{|c|}{$\begin{array}{l}\text { Hospitales } \\
\text { Públicos }\end{array}$} & \multirow{2}{*}{$\begin{array}{c}\text { Hospitales } \\
\text { Privados }\end{array}$} & \multicolumn{2}{|c|}{ Total } \\
\hline & $\mathrm{N}^{\mathrm{o}}$ & $\%$ & & $\%$ & $\mathrm{~N}^{\circ}$ \\
\hline Pacientes atendidos según tipo de hospital & 278 & 100 & 278 & 100 & 556 \\
\hline Distribución por sexo & $\mathrm{N}$ & $\%$ & $\mathrm{~N}$ & $\%$ & $\mathrm{~N}^{\circ}$ \\
\hline Femenino & 208 & 74,8 & 199 & 71,5 & 407 \\
\hline Masculino & 70 & 25,1 & 79 & 28,4 & 149 \\
\hline Distribución según grupo etario & $\mathrm{N}^{\circ}$ & $\%$ & $\mathrm{~N}$ & $\%$ & $\mathrm{~N}^{\circ}$ \\
\hline$<15$ años & 6 & 2,1 & 6 & 2,1 & 12 \\
\hline $16-25$ años & 52 & 18,7 & 30 & 10,7 & 82 \\
\hline $26-45$ años & 129 & 46,4 & 128 & 46,0 & 257 \\
\hline $46-65$ años & 75 & 26,9 & 101 & 36,3 & 176 \\
\hline$>66$ años & 16 & 5,7 & 13 & 4,6 & 29 \\
\hline Distribución de Pacientes estudiados por especialidad & $\mathrm{N}^{\circ}$ & $\%$ & $\mathrm{~N}$ & $\%$ & $\mathrm{~N}^{\circ}$ \\
\hline Medicina Interna & 59 & 21,2 & 55 & 19,7 & 114 \\
\hline Pediatría & 57 & 20,5 & 55 & 19,7 & 112 \\
\hline Cirugía General & 59 & 21,2 & 57 & 20,5 & 116 \\
\hline Cirugía Traumatológica & 56 & 20,1 & 55 & 19,7 & 111 \\
\hline Nefrología & 47 & 16,9 & 56 & 20,1 & 103 \\
\hline Nivel educativo promedio & $\mathrm{N}^{\circ}$ & $\%$ & $\mathrm{~N}$ & $\%$ & $\mathrm{~N}^{\circ}$ \\
\hline Analfabeta & 35 & 12,59 & 49 & 17,63 & 84 \\
\hline Primaria & 113 & 40,65 & 41 & 14,75 & 154 \\
\hline Secundaria & 87 & 31,29 & 80 & 28,78 & 167 \\
\hline Téenico/Universitarios & 22 & 7,91 & 46 & 16,55 & 68 \\
\hline Universitarios Superiores & 21 & 7,55 & 62 & 22,30 & 83 \\
\hline
\end{tabular}

comial (26,30\%), Diagnostico $(14,10 \%)$, Procedimiento (10,95\%), Medicación (10,95\%). Es interesante destacar que con respecto a los fallos en los "Cuidados" existe una diferencia entre las frecuencias de los HP $(33,9 \%)$ y HPR $(27,5 \%)$ de $8,5 \%$ y para la "Infección Nosocomial" de 7,4\% representando más del doble de las evidenciadas por el resto de fallos los cuales muestran diferencias que van de 1,1\% (Mixtos) a 3,1\% (Medicación).

Proceso de atención: no se evidencian diferencias significativas entre las frecuencias encontradas, sin embargo, en términos de la descripción, es preciso indicar que independientemente del tipo de entidad hospitalaria, los momentos donde se presentaron mayores casos de EA fueron: -Previo a la admisión a la sala de hospitalización $(22,5 \%)$, o al alta $(29,7 \%)$. El resto de las etapas exhibieron frecuencias inferiores al $13 \%$ y la menor frecuencia fue "durante la asistencia inmediata" $(4,3 \%)$, (Tabla III).

Con referencia a las Unidades asistenciales, en los HP se identificó mayor frecuencia de EA en las unidades de Medicina Interna y Cirugía (20,6\% en cada una), se-

Vol. 60(4): 296 - 309, 2019 


\section{TABLA II}

CARACTERIZACIÓN DE LOS EVENTOS ADVERSOS HOSPITALARIOS.

\begin{tabular}{|c|c|c|c|c|}
\hline \multirow{2}{*}{\multicolumn{2}{|c|}{ VARIABLES }} & \multirow{3}{*}{$\begin{array}{c}\mathrm{HPu} \\
\% \\
(\mathrm{~N})\end{array}$} & \multirow{3}{*}{$\begin{array}{c}\text { Hpri } \\
\% \\
(\mathrm{~N})\end{array}$} & \multirow{3}{*}{$\begin{array}{c}\text { Total } \\
\% \\
\text { (N) }\end{array}$} \\
\hline & & & & \\
\hline \multirow{2}{*}{\multicolumn{2}{|c|}{ FRECUENCIA DEL EA }} & & & \\
\hline & & 56,9 & 43 & 100 \\
\hline & Total: & $(53)$ & $(40)$ & 93 \\
\hline & $\mathrm{p}=$ & $\mathrm{N} / \mathrm{S}$ & $\mathrm{N} / \mathrm{S}$ & \\
\hline \multirow{10}{*}{$\begin{array}{l}\text { POSIBILIDAD DE } \\
\text { PREVENCIÓN DEL EA }\end{array}$} & \multirow{2}{*}{ Ligera Posibilidad } & 3,2 & 0 & 3,2 \\
\hline & & (3) & $(0)$ & 3 \\
\hline & \multirow{2}{*}{ Moderada Posibilidad } & 10,7 & 5,3 & 16 \\
\hline & & (10) & (5) & 15 \\
\hline & \multirow{2}{*}{ Elevada Posibilidad } & 27,9 & 13,9 & 41,8 \\
\hline & & $(26)$ & (13) & 39 \\
\hline & \multirow{2}{*}{ Total Evidencia de prevención } & 15 & 23,6 & 38,6 \\
\hline & & (14) & (22) & 36 \\
\hline & Total: & (53) & $(40)$ & 93 \\
\hline & $\mathrm{p}=$ & 0,02 & 0,02 & \\
\hline \multirow{16}{*}{ TIPO DE EA } & \multirow{2}{*}{ Cuidados } & 16,1 & 11,8 & 27,9 \\
\hline & & (15) & (11) & 26 \\
\hline & \multirow{2}{*}{ Infección Nosocomial } & 10,7 & 10,7 & 21,4 \\
\hline & & (10) & (10) & 20 \\
\hline & \multirow{2}{*}{ Procedimiento } & 5,3 & 3,2 & 8,5 \\
\hline & & (5) & (3) & 8 \\
\hline & \multirow{2}{*}{ Medicación } & 5,3 & 5,3 & 10,6 \\
\hline & & (5) & $(5)$ & 10 \\
\hline & \multirow{2}{*}{ Diaǵnostico } & 6,4 & 4,3 & 10,7 \\
\hline & & (6) & (4) & 10 \\
\hline & \multirow{2}{*}{ Otros } & 2,1 & 1 & 3,1 \\
\hline & & (2) & (1) & 3 \\
\hline & \multirow{2}{*}{ Mixtos } & 10,7 & 6,4 & 17,1 \\
\hline & & (10) & (6) & 16 \\
\hline & Total & (53) & (40) & 93 \\
\hline & $\mathrm{p}=$ & $\mathrm{N} / \mathrm{S}$ & $\mathrm{N} / \mathrm{S}$ & \\
\hline
\end{tabular}

HPu: Hospitales Públicos, HPr: Hospitales Privados. 
TABLA III

PERÍODO DE HOSPITALIZACIÓN DONDE OCURRIÓ EL EA.

\begin{tabular}{cccc}
\hline & & HPu & Hpri \\
\hline & Previo a la Admisión & 9,6 & 12,9 \\
& & $(9)$ & $(12)$ \\
& Al momento de la admisión & 13,9 & 5,3 \\
PERÍODO DE HOSPITALIZACIÓN & & $(7)$ & $(5)$ \\
DONDE OCURRIÓ EL EA & Procedimiento & 10,7 & 1 \\
& Durante la asistencia Inmediata & $(10)$ & $(1)$ \\
& Asistencia en sala & $12)$ & 2,1 \\
& & $(16)$ & $(2)$ \\
& Al alta & 17,9 & 11,8 \\
& Total: & $(9)$ & $(11)$ \\
& $\mathrm{p}=$ & $(53)$ & $(40)$ \\
& & $\mathrm{N} / \mathrm{S}$ & $\mathrm{N} / \mathrm{S}$ \\
\hline
\end{tabular}

HPu: Hospitales Públicos, HPr: Hospitales Privados.

guidas de Pediatría (22,5\%) (Tabla IV). En los hospitales privados, los hallazgos fueron levemente diferentes, al prevalecer los EA en la Unidad de Medicina Interna $(27,5 \%)$, seguida de Pediatría $(22,5 \%)$ y luego otras unidades (35\%), (Tabla IV).

Referente al nivel de gravedad con que se manifestaron los EA, los de mayor frecuencia tanto en HP como en HPR fueron los de gravedad media, con frecuencias de $31,7 \%$ y $\mathbf{5 2 , 5 \%}$, respectivamente (Tabla IV). En los HP, la distribución de EA de gravedad media, fue homogénea entre todas las unidades asistenciales, con frecuencias que iban desde 9,4\% para Cirugía, hasta 1,8\% en Nefrología - Urología; por otro lado, la distribución en los HPR se concentró principalmente en "Otros Departamentos" (25\%) y Pediatría (15\%). Los EA de gravedad alta ocurrieron en la Unidad de Cirugía (11,3\%) $(\mathrm{N}=6)$, y en servicios distintos a las unidades clínicas $(9,4 \%)(\mathrm{N}=5)$.

En el caso de los hospitales privados, los EA de gravedad alta se presentaron en su mayoría en la Unidad de medicina interna $(17,5 \%)(\mathrm{N}=7)$. Finalmente, los EA de gravedad leve (Tasa global: $\mathrm{N}=28$ 30,1\%) ocurren principalmente en los hospitales públicos
$(\mathrm{N}=21 / 39,2 \%)$ (Tabla IV). En este estudio no se identificaron casos de EA que sugirieran relación con algún fallecimiento, o casos de defunciones por algún EA específico.

La aparición de EA de gravedad media y alta, tanto en hospitales públicos como en privados, de acuerdo al análisis de correspondencia, estuvieron relacionados con falla en los cuidados hospitalarios $(\mathrm{p}<0,05)$, errores de diagnóstico y fallos en la medicación $(\mathrm{p}<0,05)$, de igual forma los factores de riesgo de tipo Cardiovascular y Endocrino metabólicos también se ven asociados.

El Impacto del EA, se ve reflejado en la estancia hospitalaria (Tabla IV), observando que, en los hospitales públicos, se incrementó en un promedio de 10 días en el 39,3\%. Por lo tanto, se detectaron 21 eventos adversos que aumentaron a 210 días la estancia hospitalaria. En los hospitales públicos se presentó un promedio de 8,3 EA en 100 días de hospitalización (95\% IC 7,4-9,2); con una tasa de reingresos de $41,2 \%(\mathrm{~N}=22)$.

En los hospitales privados, la hospitalización promedio de un paciente es de 5 días; si este padece un EA, su estancia se incrementa 10 días más (15 días promedio de hospitalización); ocurriendo esto en el

Vol. 60(4): 296 - 309, 2019 
TABLA IV

GRAVEDAD DEL EA SEGÚN TIPO DE HOSPITAL Y UNIDAD ASISTENCIAL.

\begin{tabular}{|c|c|c|c|c|c|c|c|c|c|c|c|c|c|c|c|c|c|c|c|}
\hline \multirow{2}{*}{\multicolumn{2}{|c|}{ VARIABLES }} & \multicolumn{9}{|c|}{ HOSPIT ALES PÚBLICOS } & \multicolumn{8}{|c|}{ HOSPITALES PRIVADOS } & \multirow[b]{2}{*}{ 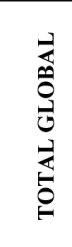 } \\
\hline & & & 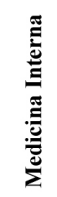 & 尊 & 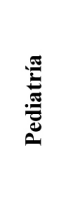 & 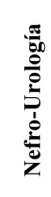 & 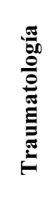 & $\stackrel{t}{0}$ & 趈 & $=$ & 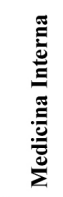 & 吾 & 吾 & 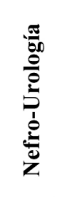 & 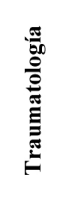 & $\stackrel{n}{\underline{z}}$ & 离 & $\approx$ & \\
\hline \multirow{8}{*}{$\begin{array}{l}\text { NIVELES DE } \\
\text { GRAVEDAD }\end{array}$} & \multirow{2}{*}{ Baja } & $\%$ & 11,3 & 3,7 & 16,9 & 3,7 & 1,8 & 1,8 & 39,2 & & 12,5 & 0 & 0 & 0 & 0 & 5,0 & 17,5 & & 30,1 \\
\hline & & $\mathbf{N}$ & 6 & 2 & 9 & 2 & 1 & 1 & 21 & & 5 & 0 & 0 & 0 & 0 & 2 & 7 & & 28 \\
\hline & \multirow{2}{*}{ Media } & $\%$ & 7,5 & 9,4 & 3,7 & 1,8 & 1,8 & 7,5 & 31,7 & 0,28 & 5,0 & 0 & 15 & 2,5 & 5,0 & 25,0 & 52,5 & 0,28 & 40,8 \\
\hline & & $\mathbf{N}$ & 4 & 5 & 2 & 1 & 1 & 4 & 17 & & 2 & 0 & 6 & 1 & 2 & 10 & 21 & & 38 \\
\hline & \multirow{2}{*}{ Alta } & $\%$ & 1,8 & 11,3 & 1,8 & 1,8 & 1,8 & 9,4 & 27,9 & & 17,5 & 2,5 & 5,0 & 0 & 5,0 & 0 & 30,0 & & 29,0 \\
\hline & & $\mathrm{N}$ & 1 & 6 & 1 & 1 & 1 & 5 & 15 & & 7 & 1 & 2 & 0 & 2 & 0 & 12 & & 27 \\
\hline & \multirow{2}{*}{ Total } & $\%$ & 20,7 & 24,5 & 22,6 & 7,5 & 5,6 & 18,8 & 100 & & 35,0 & 3 & 20,0 & 3 & 10,0 & 30,0 & 100 & & 100,0 \\
\hline & & $\mathrm{N}$ & 11 & 13 & 12 & 4 & 3 & 10 & 53 & & 7 & 1 & 2 & 0 & 2 & 2 & 40 & & 93,0 \\
\hline \multirow{8}{*}{$\begin{array}{l}\text { REPERCUSIÓN } \\
\text { DEL EA EN LA } \\
\text { ESTANCIA }\end{array}$} & \multirow{2}{*}{$\begin{array}{l}\text { No Au- } \\
\text { mentó la } \\
\text { estancia }\end{array}$} & $\%$ & 5,6 & $\mathbf{0}$ & 7,5 & $\mathbf{0}$ & 1,8 & 3,7 & 18,6 & & 0 & 0 & 2,5 & 0 & 0 & 10 & 12,5 & & 16,1 \\
\hline & & $\mathbf{N}$ & 3 & 0 & 4 & 0 & 1 & 2 & 10 & & 0 & 0 & 1 & 0 & 0 & 4 & 5 & & 15 \\
\hline & \multirow{2}{*}{$\begin{array}{c}\text { Aumentó } \\
\text { Parte de } \\
\text { la estancia }\end{array}$} & $\%$ & 11,3 & 3,7 & 5,6 & 5,6 & 3,7 & 9,4 & 39,3 & 0,2 & 10,0 & 0 & 0 & 2,5 & 10,0 & 15 & 37,5 & 0,2 & 38,7 \\
\hline & & $\mathbf{N}$ & 6 & 2 & 3 & 3 & 2 & 5 & 21 & & 4 & 0 & 0 & 1 & 4 & 6 & 15 & & 36 \\
\hline & \multirow{2}{*}{$\begin{array}{l}\text { Causó un } \\
\text { reingreso }\end{array}$} & $\%$ & 3,7 & 16,9 & 9,4 & 1,8 & $\mathbf{0}$ & 9,4 & 41,2 & & 17,5 & 2,5 & 20,0 & 0 & 0 & 10,0 & 50,0 & & 45,0 \\
\hline & & $\mathrm{N}$ & 2 & 9 & 5 & 1 & 0 & 5 & 22 & & 7 & 1 & 8 & 0 & 0 & 4 & 20 & & 42 \\
\hline & \multirow{2}{*}{ Total } & $\%$ & 20,6 & 20,6 & 22,5 & 7,4 & 5,5 & 22,5 & 100 & & 27,5 & 2,5 & 22,5 & 2,5 & 10 & 35 & 100 & & 100 \\
\hline & & $\mathbf{N}$ & 11 & 11 & 12 & 4 & 3 & 15 & 53 & & 11 & 1 & 9 & 1 & 4 & 14 & 40 & & 93 \\
\hline
\end{tabular}

$37,5 \%(\mathrm{~N}=15)$ de los pacientes. Las instituciones privadas presentan una tasa de 25 EA por cada 100 días de hospitalización (IC: $95 \% 24-26$ ), con un promedio de reingresos del 50\% (Tabla IV).

Referente al impacto de las lesiones causadas por los EA, no hubo diferencias estadísticas entre hospitales públicos y privados. Por otro lado, el 50,5\% de los EA detectados ocasionaron una severa incapacidad $(\mathrm{N}=47)$, en los pacientes (Tabla V), e incapacidad absoluta en el $25,8 \%$ de los casos $(\mathrm{N}=24)$.

\section{DISGUSIÓN}

Los resultados obtenidos permiten evidenciar que la prevalencia estimada de 16,5\% de EA determinada en las instituciones estudiadas supera el 10\% de la tasa global de países latinoamericanos planteada en otros estudios $(5,23)$; al igual que la tasa de evitabilidad superior al $80 \%$, que también prevalece el 60\% del IBEAS y de otros países (23). Aunque estas cifras podrían atribuirse a la similitud metodológica con los estudios referentes y su sensibilidad en la detección de EA (17) también consideramos fundamental la particularidad y realidad de cada hospital y el estudio en conjunto (23).

En consideración con los hospitales públicos y privados, las literatura no respalda que los hospitales del sector privado suelen ser más eficientes o médicamente efectivos que el sector público (29); hecho observado en esta investigación; lo que nos invita a reflexionar sobre los elementos comunes entre las dos categorías de institución, más allá del componente económico, pudiendo estar asociados al nivel de compromiso de la 


\begin{tabular}{cccc}
\multicolumn{4}{c}{ TABLA V } \\
INCAPACIDAD PROVOCADA POR EL EVENTO \\
ADVERSO. \\
\hline Deterioro & $\mathrm{HPu}$ & $\mathrm{HPr}$ & Total \\
Físico & $\%$ & $\%$ & $\%$ \\
& $\mathrm{~N}$ & $\mathrm{~N}$ & $\mathrm{~N}$ \\
\hline Leve o Ninguna & 24,5 & 22,5 & 23,6 \\
Incapacidad & 13 & 9 & 22 \\
Severa & 47,1 & $\mathbf{5 5}$ & $\mathbf{5 0 , 5}$ \\
Incapacidad & 25 & 22 & 47 \\
Incapacidad & 28,3 & 22,5 & 25,8 \\
Absoluta & 15 & 9 & 24 \\
Relacionado con & 0 & 0 & 0 \\
el fallecimiento & 0 & 0 & 0 \\
Fallecimiento & 0 & 0 & 0 \\
Total & 0 & 0 & 0 \\
p= & $\mathbf{5 3}$ & 40 & 93 \\
\hline
\end{tabular}

HPu: Hospitales Públicos, HPr: Hospitales Privados

cultura de calidad y seguridad del paciente dentro de las instituciones y las personas al cuidado de los enfermos $(17,34)$. Otro factor en este resultado, es la influencia del método aleatorizado, en el que los hospitales seleccionados tuvieron una forma de trabajo similar entre públicos y privados, garantizando la idoneidad en la captación del EA y la validez de identificación de su carácter iatrogénico y prevenible.

Este estudio, es uno de los pocos que valora los EA, desde el análisis comparativo en instituciones de gestión de salud pública y privada (35) haciendo un nuevo aporte teórico, ya que este es el modelo de asistencia existente en la mayoría de los países de América y Europa $(36,37)$.

De la presente investigación, podemos deducir que los elementos que caracterizaron a los EA detectados, estuvieron asociados en primer lugar al tipo de Unidad asistencial donde se presentaron, que en este estudio fueron las Unidades de Medicina Interna y las Unidades Quirúrgicas las que tuvieron la mayor ocurrencia de EA y de gravedad alta, resultados que son similares a los de otras experiencias investigativas, $\mathrm{y}$ atribuidos a que en estas áreas, debido a su naturaleza, la exposición a los riesgos es mayor, y al nivel de interacción "necesaria" con profesionales de la línea directa de atención $(9,10,13,14,17)$. Los equipos de trabajo en las unidades asistenciales y su nivel de compromiso con la seguridad del paciente, cumplen un rol tan importante, que el impacto de los EA, se ve reflejado en la estancia hospitalaria en estas unidades, aumentando esta o causando un reingreso, que como observamos se expresa en este estudio en una cifra considerable; así como la severa incapacidad física que ocasionaron los EA en un numeroso grupo de pacientes cuyo período de recuperación es mayor al previsto por su enfermedad.

Otro factor asociado al riesgo de ocurrencia es el período de la asistencia donde ocurre el EA, la cual define qué aspecto del proceso individual de atención, estuvo involucrado en la ocurrencia del daño; los extremos de la atención (admisión a hospitalización y alta), son los más frecuentes en la prevalencia de EA en esta área $(3,17,38)$. Sin embargo, en los hospitales privados la ocurrencia se establece en cualquier momento de la atención, mientras que en los públicos suceden con más frecuencia en la "asistencia en sala"; en este sentido Michel y col. (12), expusieron claramente que los riesgos están asociados a los cuidados pre-operatorios y Zegers y col. (14) concluyeron que principalmente durante la admisión, considerado este momento de la atención donde la comunicación entre el paciente y el profesional de atención directa, es cuando se ejerce un rol fundamental en la minimización de riesgos de EA (14).

La naturaleza o tipología del EA, también representa un elemento relacionado a su ocurrencia, ya que la literatura documenta que las infecciones asociadas a la atención en salud (IAAS) es el EA más frecuente $(6,9,17)$ siendo este estudio una muestra de ello. En este sentido, cabe resaltar un hecho aunque teóricamente conocido (39), pero 
poco documentado, en donde los EA más severos o graves se asocian a una combinación de EA de distinta naturaleza, es decir, no solo la infección nosocomial, en este caso como la más frecuente, sino que se suman errores de procedimiento, diaǵnóstico y cuidados, lo que ocasionan EA de gravedad alta, generando principalmente una incapacidad severa y/o absoluta como impacto del EA, y por supuesto mayor estancia hospitalaria, lo que aumenta la posibilidad de infeceiones adquiridas en el hospital y el incremento de los costos en salud, entre otras $(6,23)$.

Basados en los resultados de cada institución, aproximadamente unos 600 pacientes ingresados en hospitalización, podrían padecer daño por error en la atención, de los cuales 30 eventos adversos podrían ocasionar lesiones graves siendo altamente prevenibles, generando severa incapacidad o incapacidad absoluta cuyo efecto es más prolongado en el tiempo, por lo tanto, el período de recuperación es mayor en el mejor de los casos.

Una de las contribuciones de este trabajo, es atender la recomendación del estudio IBEAS, en el que sugiere adaptar la Guía de Cribado a las condiciones locales, lo cual permitió mejorar el conocimiento de las circunstancias en que se producen los EA sin alterar el resultado de su ocurrencia (17); orientando estos resultados a implementar herramientas sobre la seguridad del paciente, para generar un impacto en la minimización del daño.

Se describen algunas limitaciones que deben ser tenidas en cuenta en la interpretación de los resultados. El diseño utilizado impide que los resultados se generalicen a la totalidad de los hospitales del país, sin embargó, reflejan la atención de un número importante de pacientes de la región. Así mismo, debido al establecimiento de los criterios de inclusión, también utilizados en otros estudios similares, existe la posibilidad de haber infrarrepresentado casos de ocurrencia de EA. El sesgo de temporalidad no tiene gran implicación, ya que el diseño del estudio no urge una premisa de precedencia temporal.

Por otro lado, siendo la historia clínica la principal fuente de datos para la identificación y prevalencia de EA, debido a la baja calidad de los registros médicos para documentar los EA, estas cifras pudiesen estar infravaloradas, sin embargó, se validó presencialmente mediante la observación directa al paciente mediante la presencia del EA o sus secuelas, lo que minimizaba de forma importante este sesgo. Sin embargo, esto no puede ser una limitación para desarrollar estos estudios, aunque sea en niveles primarios como es el caso, ya que estos son la base para estudios más amplios y a gran escala.

Finalmente, la literatura plantea la posibilidad de un sesgo de supervivencia $(18,40)$, debido a que los EA podrían verse sobrerrepresentados en varios escenarios; en este sentido dos situaciones a favor nos presenta el diseño del estudio: 1.- Durante la fase de cribado, el revisor primario previamente entrenado observó al paciente y validaba la presencia o secuela del EA, lo cual minimiza el sesgo por EA difícilmente identificables y por infección nosocomial. 2.- La tasa de EA que causaron un reingreso, descrita anteriormente, es un elemento que fue determinado durante la revisión de la historia clínica. Durante la fase de cribado, el primer momento para identificar los EA es cuando este ocurrió "durante el ingreso a sala de hospitalización" aunque haya sido identificado durante el período de estudio, por lo tanto, mediante un método de observación activa, cuando se identificaba un paciente con un reingreso por esta naturaleza, este no era incluido en el estudio, lo cual disminuye la posibilidad de que los EA que ocasionen un reingreso se vean sobrerrepresentados.

En relación a la fiabilidad de los datos obtenidos, y aunque no se realizó una prueba de concordancia entre evaluadores, la capacitación de los encuestadores y revisores descrita previamente, y los métodos de vigilancia activa reducen al máximo la posibilidad de este sesgo. 
La presente investigación sugiere diversas implicaciones en el contexto actual y futuro del sector Salud en Venezuela, sirviendo como referencia para tomar acciones estratégicas e institucionales, articulando ambos sectores públicos y privados y ubicando al Zulia como referente de Venezuela en el mapa de la Seguridad del paciente en el país y en Latinoamérica, con sistemas de salud similares.

A modo concluyente, aunque los resultados de la investigación responden a una prevalencia marcadamente superior al promedio en estudios Latinoamericanos, no se puede concluir que en Venezuela hay una frecuencia mayor, dada las diferencias metodológicas. Harían falta futuros estudios comparativos donde se pueda plantear el estudio de EA de manera sistemática y con un sistema similar al de viǵilancia epidemiológica homogéneo. Los resultados obtenidos mostraron que el paciente se hace más vulnerable a padecer un EA al principio y al final de la atención, así como el tipo de unidad asistencial, por lo que requiere atender con estrategias de implementación de protocolos aplicados por el profesional de la salud o programas educativos de inclusión al paciente en su proceso, para que todos aborden la sistemática del proceso y blindarlo. Esta información busca sustentar un planteamiento estratégico de acción ǵlobal y en red entre las instituciones, el personal de salud y los pacientes para garantizar seguridad en la atención en salud.

\section{REFERENCIAS}

1. Kohn LT, Corrigan JM, Donaldson, Molla S E. To Err is Human [Internet]. To Err Is Human: Building a Safer Health System. Washington, DC: National Academy Press; 1999. 15-312 p.

2. Jim S. Building a safer NHS for patients: Implementing an Organisation with a Memory [Internet]. Department of Health. England; 2004. Available from: http://www. femeba.org.ar/fundacion/
3. WHO. Seguridad del paciente Informe de la Secretaría y la calidad de la atención de salud. [Internet]. World Alliance for Patient Safety. Ginebra; 2006. Available from: http://apps.who.int

4. Shekelle P, Pronovost P, Wachter R, McDonald K, Schoelles K, Dy S, Shojania K, Reston J, Adams A, Angood P, Bates D, Bickman L, Carayon P, Donaldson SL, Donna N Trisha, Greenhalgh T, Haughom J, Lake E, Richard K, Meyer G, Miller M, Neuhauser D, Ryan G, Saint S, Shortell S, Stevens D, Walshe K. The top patient safety strategies that can be encouraged for adoption now. Ann Intern Med 2012;158(5):2011-2012.

5. Aranaz J, Aibar C, Limón R, Amarilla A, Restrepo F, Urroz O, Sarabia O, García L, Terol E, Agra Y, Gonseth J, Bates D. Prevalence of adverse events in the hospitals of five Latin American countries: results of the 'Iberoamerican study of adverse events (IBEAS). BMJ Qual Saf [Internet]. 2011;20(May):1043-1052.

6. World Alliance for Patient Safety. Summary of the evidence on patient safety: Implications for research. [Internet]. Ginebra; 2008. Available from: http://www.who.int

7. Brennan TA, Leape LL, Laird NM, Hebert L, Localio AR, Lawthers AG, Newhouse JP, Weiler PC, Hiatt HH. Incidence of adverse events and neǵligence in hospitalized patients. Results of the Harvard Medical Practice Study I. N Enǵl J Med 1991;324(6):370-376.

8. Thomas EJ1, Studdert DM, Burstin HR, Orav EJ, Zeena T, Williams EJ, Howard KM, Weiler PC BT. Incidence and types of adverse events and neǵligent care in Utah and Colorado. Med Care 2000;38(3):261271.

9. Schiøler T, Lipezak H, Pedersen BL, Mogensen TS, Bech KB, Stockmarr A, Svenning AR, Frolich A. [Incidence of adverse events in hospitals. A retrospective study of medical records]. Ugeskr Laeger 2001;163(39):5370-5378.

10. Baker GR, Norton PG, Flintoft V, Blais R, Brown A, Cox J, Etchells E, Ghali WA, Hébert P, Majumdar SR, O'Beirne M, Palacios-Derflingher L, Reid RJ, Sheps S, Tamblyn R. The Canadian Adverse Events Study: the incidence of adverse events

Vol. 60(4): 296 - 309, 2019 
among hospital patients in Canada. CMAJ 2004;170(11):1678-1686.

11. Vincent $\mathrm{C}$, Neale G, Woloshynowych $M$. Adverse events in British hospitals: preliminary retrospective record review. BMJ 2001;322(7285):517-519.

12. Michel P, Quenon JL, Djihoud A, TricaudVialle S, De Sarasqueta AM. French national survey of inpatient adverse events prospectively assessed with ward staff. Qual Saf Heal Care 2007;16(5):369-377.

13. Aranaz J, Aibar C, Vitaller J, Ruiz P, Limón $\mathbf{R}$, Terol E. Incidence of adverse events related to health care in Spain: Results of the Spanish National Study of Adverse Events. J Epidemiol Community Health 2008;62(12):1022-1029.

14. Zegers M, De Bruijne MC, Wagner C, Hoonhout LHF, Waaijman R, Smits M, Hout FA, Zwaan L, Christiaans-Dingelhoff I,Timmermans DR, Groenwegen PP, Van der Wal G. Adverse events and potentially preventable deaths in Dutch hospitals: Results of a retrospective patient record review study. Qual Saf Heal Care 2009;18(4):297302.

15. Rafter N, Hickey A, Conroy RM, Condell S, Connor PO, Vaughan D, Walsh G, Williams D. The Irish National Adverse Events Study ( INAES ): the frequency and nature of adverse events in Irish hospitals - a retrospective record review study. BMJ Qual Saf2017;26(2):111-119.

16. Sommella L, Waure C De, Ferriero AM, Biasco A, Mainelli MT, Pinnarelli L, Ricciardi W, Damiani G. The incidence of adverse events in an Italian acute care hospital : findings of a two-stage method in a retrospective cohort study. BMC Health Serv Res 2014;14(358):1-8.

17. Aranaz JM, Aibar C. Estudio IBEAS Prevalencia de efectos adversos en hospitales de Latinoamérica [Internet]. Ocsavinfo. 2010. p. 168. Available from: http://www.seguridaddelpaciente.es/ contenidos/castellano/2009/INFORME_IBEAS.pdf?phpMyAdmin = mvRY xVABNPM34i7Fnm,23Wrlq5

18. Aranaz J, Aibar C, Limón R, Restrepo F, Urroze O, Sarabiaf $R$, Ingag $R$, Santivañezh A , Larizgoitia I y ET-G. Diseño del estudio IBEAS: prevalencia de efectos ad- versos en hospitales de Latinoamérica. Rev Calid Asist 2011;26(3):194-200.

19. Ministerio de Salud y Protección Social. Decreto 1011 de 2006: Sistema Obliǵatorio de Garantía de Calidad de la Atención de Salud. Decreto [Internet]. 2006;17. Available from: http://www.alcaldiabogota.gov. co/sisjur/normas/Norma1.jsp?i=19975\#0

20. Ministerio de salud; subsecretaría de salud pública. Resolución 1031 exenta. [Internet]. Chile; 2016 p. 121-127. Available from: Ministerio de salud; subsecretaría de salud pública.

21. Congreso de la República. Ley del Ministerio de Salud N² 27657. 2002;1-16. Available from: http://www.digemid.minsa.gob.pe/ UpLoad/UpLoaded/PDF/LEYN27657.pdf

22. Mendes W. The assessment of adverse events in hospitals in Brazil. Int J Qual Health Care. 2009;21(4):279-284.

23. Mendes W, Luiza A, Pavão B, Martins M, Travassos C. The application of Iberoamerican study of adverse events ( IBEAS ) methodology in Brazilian hospitals. Int J Qual Heal Care 2018;30(6):480-485.

24. Asamblea Nacional de la Republica Bolivariana de Venezuela. Ley Orgánica del Sistema Venezolano para la Calidad. 37.555 Venezuela; p. 2-39. Available from: http:// www.sencamer.gob.ve

25. Gaceta Oficial de la República Bolivariana de Venezuela $\mathrm{N}^{\circ}$ 246.948. Normas sobre clasificación de establecimientos de atención médica del subsector público. 1983.

26. Rivas P, Alonso G. Regulación de la dispensación de medicamentos y su efecto en el consumo de antibióticos en Venezuela. Rev Panam Salud Pública 2012;30(6):592-597.

27. Instituto Nacional de Estadística. XIV Censo nacional de población y vivienda ResultadosporEntidadFederalyMunicipio delEstadoZulia. [Internet]. 2014. Available from: ww.ine.gov.ve/documentos/Demografia/ CensodePoblacionyVivienda/pdf/zulia.pdf

28. OPS Organización Panamericana de la Salud. Análisis comparado del marco jurídico sobre calidad de la atención y seguridad del paciente (CASP) en América Latina , España y la Comunidad Europea [Internet]. Washington: WHO; 2012; 1-126. Available from: https://apps.who.int/iris/handle/10665/310372 
29. Basu S, Andrews J, Kishore S, Panjabi R, Stuckler D. Comparative performance of private and public healtheare systems in low- and middle-income countries: A systematic review. PLoS Med 2012;9(6):19.

30. Ministerio del Poder Popular para la salud. Normas para la clasificación de establecimientos asistenciales del sistema público nacional de salud. 2014.

31. López C, Santin D. Minería de datos: técnicas y herramientas. Paraninfo T, editor. Madrid: Editorial Paraninfo, 2007. p. 101; 6-808 p.

32. Proyecto IDEA. Estudio de la incidencia de efectos adversos en la asistencia hospitalaria. [Internet]. 2004. Available from: http://www.dsp.umh.es/proyectos/idea/ index.html.

33. Proyecto IDEA. Cuestionario Modular (MRF2) para identificación de sucesos adversos por revisión de historias clínicas. [Internet]. 2004. Available from: http://www. who.int/patientsafety/research/ibeas mrf2_sp.pdf.

34. Irfan SM, Ijaz A. Comparison of Service Quality Between Private and Public Hospitals : Empirical Evidences From Pakistan. J Qual Technol Manag 2011;VII(I):1-22.

35. Aljadhey H, Mahmoud MA, Ahmed Y, Sultana R, Zouein S, Alshanawani S, Mayet A, Alshaikh M, Kalaǵi N, Al Tawil E, Rahman A, Arwadi A, Alyahya M, Murray M, Bates D. Incidence of adverse drug events in public and private hospitals in Riyadh, Saudi Arabia: the ( ADESA ) prospective cohort study. BMJ Open 2016;6(7):1-8.
36. Marino J. Gonzalez R. Reformas del Sistema de Salud en Venezuela (1987-1999):balance y perspectivas [Internet]. CEPALECLAC Naciones Unidas, editor. Santiaǵo de Chile; 2001; 19-33.

37. Ministerio de Sanidad SS e I. Los sistemas sanitarios en los países de la Unión Europea. 2017. Available from: http://www. mscbs.gob.es.

38. Mostaza J, Muinelo I, Cristian T. Prevalencia y gravedad de efectos adversos durante la hospitalización. Med Clin (Barc) 2005;124(2):0-80. Available from: https:// www.elsevier.es/es-revista-medicina-clinica2-articulo-prevalencia-gravedad-efectos-adversos-durante-13070462.

39. World Health Organisation (WHO). Marco Conceptual de la Clasificación Internacional para la Seguridad del Paciente Informe Técnico Definitivo Enero de 2009 [Internet]. 2009. Available from: http://www. who.int/patientsafety/implementation/ icps/icps_full_report_es.pdf

40. Matarán E, Aǵuilar R, Muñoz M. Incidencia y tipo de efectos adversos durante el procedimiento de hemodiálisis. Enferm Nefrol 2013;16(1):36-40. 